\title{
Metabolomics Methods as a New Diagnostic Tool for Thyroid Nodules
}

\author{
Wojtowicz W' $\mathbf{W}^{1}$, Pawełka $\mathbf{D}^{2}$, Balcerzak $\mathbf{W}^{2}$ and Młynarz $\mathbf{P}^{1^{*}}$ \\ ${ }^{1}$ Bioorganic Chemistry Group, Department of Chemistry, Wroclaw University of Technology, 50-370 Wroclaw, Poland \\ ${ }^{2}$ First Department and Clinic of General, Gastroenterological and Endocrinological Surgery, Wrocław, Poland
}

\begin{abstract}
Carcinoma of the thyroid gland is the most common cancer of the endocrine system. It accounts for approximately $10 \%$ of thyroid focal lesions, and the incidence of this cancer is increasing. A valuable technique for differentiating cancerous from benign nodules is fine-needle aspiration biopsy (FNA) with cytological verification. Unfortunately, in $30 \%$ of cases, FNA results are not sufficient to determine the proper method of treatment. Therefore, many patients are referred for diagnostic surgery and histopathologic examination. Despite the development of new imaging and molecular diagnostic techniques, no universal marker for pre-surgery identification of malignant changes in the thyroid is available.

Modern measuring techniques, such as nuclear magnetic resonance spectroscopy - NMR - and mass spectrometry - MS, in combination with chemometric analysis led to the development of a new field of biology - metabolomics. Metabolomics allows for the analysis of biochemical processes in biological systems by assessing the metabolome (set of all metabolites - small molecular compounds with a molecular weight $<1000 \mathrm{Da}$ - contained in measured biological material). The metabolite profile quantitatively and qualitatively changes in response to disturbances of homeostasis. The promising results of the use of metabolomics methods as diagnostic tools for certain cancers and our experience with using metabolomics methods to differentiate benign from malignant thyroid tumors suggest that this field of science, which has been growing for several years, will improve the diagnosis and differentiation of thyroid cancer.
\end{abstract}

Keywords: Thyroid nodules; Thyroid; Metabolomics; Personalized medicine; Biomarker

\section{Introduction}

Focal thyroid lesions in the form of individual nodules or multinodular goiters are one of the most common problems for endocrinologists and endocrine surgeons. Available sources show that they affect a large population of patients. They are identified by palpation in $2-6 \%$ of cases, by ultrasound in $19-35 \%$ of cases and by autopsy in $8-65 \%$ of cases [1]. It is estimated that approximately $4-12 \%$ of thyroid lesions are cancerous, with most being papillary carcinoma lesions [2]. In recent decades, the morbidity of thyroid cancer has increased in both women and men [3-5]. Large tumors with compression symptoms and quickly growing tumors clearly require surgery. Wide access to imaging diagnostics has made it possible to frequently detect small nodules in the thyroid parenchyma in patients without clinical symptoms. A commonly adopted diagnostic and therapeutic strategy sequence is the following: ultrasound - fine-needle biopsy under ultrasound control - decision to perform surgery on the basis of cytology or active surveillance.

Ultrasound tumor characteristics indicating a greater risk of cancer (macro- and micro-calcifications, solid character of the tumor, irregular boundaries, central tumor vasculature, absence of a hypoechoic capsule, greater longitudinal than transverse dimension) do not affect the final diagnosis [6-9]. Other thyroid imaging methods, like computed tomography, standard magnetic resonance imaging and positron emission tomography (PET) are not better at differentiating benign and malignant lesions than ultrasound. There is hope that a new ultrasound technique - elastography - will be better at evaluating the cohesiveness of the tumor and, thus, will better indicate the risk of malignant changes $[10,11]$.

The golden standard for the diagnosis of thyroid nodules is fine-needle biopsy under ultrasound control. The Bethesda system was introduced in 2007 by the National Cancer Institute (NCI) to describe cytopathology of thyroid tumors, and it divides them into 6 categories: nondiagnostic or unsatisfactory (I), benign (II), atypia of undetermined significance (AUS) or follicular lesion of undetermined significance (FLUS) (III), follicular neoplasm (FN) or suspicious for FN (IV), suspicious for malignancy (V), and malignant (VI) [12]. The risk of malignant changes for each of these groups is as follows: I- 1-4\%, II- 0-3\%, III- 5-15\%, IV- 15-30\%, V- 60-75\%, and VI- 97-99\%. AUS, FLUS, FN and suspicion of malignancy are recognized in $5-42 \%$ of cases and act together to form an indeterminate diagnosis; they are all indications for diagnostic surgery $[6,13,14]$.

The procedure implemented in cases of AUS/FLUS has been widely discussed. Re-biopsy, which is recommended, sometimes changes the diagnosis and helps confirm the decision to perform surgery. For some patients, obtaining multiple biopsies of the same tumor is necessary. The diagnosis of malignancy by re-biopsy delays the implementation of radical surgical treatment of the cancer, and in some cases, exclusion of cancer is not possible without the performance of lobectomy or thyroidectomy [15,16]. Discovering non-cytological markers of malignancy seems particularly important for this category of patients in order to reduce the performance of unnecessary operations, allow for execution of the most tissue-conserving surgery possible and achieve accurate diagnoses allowing for appropriate therapeutic choices to prevent the need for re-peat operations [17].

There are great expectations in genetic research. The development of molecular biology and the possibility of its use in medicine have resulted in the performance of a series of studies on cancer genetics,

*Corresponding author: Młynarz P, D.Sc, Bioorganic Chemistry Group, Department of Chemistry, Wroclaw University of Technology, 50-370 Wroclaw, Poland, Tel: +48 07132045 97, 606898 616; E-mail: piotr.mlynarz@pwr.edu.pl

Received December 21, 2015; Accepted January 11, 2016; Published January 13, 2016

Citation: Wojtowicz W, Pawełka D, Balcerzak W, Młynarz P (2016) Metabolomics Methods as a New Diagnostic Tool for Thyroid Nodules. Metabolomics 6: 161. doi:10.4172/2153-0769.1000161

Copyright: (c) 2016 Wojtowicz W, et al. This is an open-access article distributed under the terms of the Creative Commons Attribution License, which permits unrestricted use, distribution, and reproduction in any medium, provided the original author and source are credited. 
including cancer of the thyroid. Important genetic changes include RAS gene mutations, the PAX8/PPARr translocation (currently more often present in cancer than in follicular adenoma) and translocation of the proto-oncogene RET/PTC in papillary carcinoma and benign lesions [6]. A new algorithm has been developed for the handling of undefined thyroid tumors, and it involves molecular tests of material obtained by fine needle aspiration as a supplement to cytology analysis (Figure 1) [17].

Xing et al. also highlighted the role of BRAF mutations in assessing the aggressiveness of papillary carcinoma, which may affect surgical treatment decisions [17].

There are also reports in the literature of using microRNA (miRNA) profiles to distinguish papillary carcinoma from normal thyroid tissue and to distinguish follicular carcinoma from follicular adenoma [6]. Reports of miRNA profiles in serum are particularly interesting, as new serum miRNA markers could be measured non-invasively. A study by Yu et al. found that a serum miRNA profile of the miRNAs miR-151-5p, miR-222 and let-7e provided $87.8 \%$ and $86.85 \%$ sensitivities and $88.8 \%$ and 79.5 specificities for the differentiation of papillary carcinoma from benign tumors and healthy controls, respectively. However, there was no significant difference in the level of let-7e between benign nodules and cancerous tissue, which may suggest that the increased serum concentration of let-7e could be due to other factors that are not necessarily related to the presence of a malignant tumor in the thyroid gland $[6,18,19]$.

Detection of increased activity of a number of genes in tumor tissues has resulted in the performance of research to identify markers of thyroid carcinogenesis based on the transcriptional products of those genes in the peripheral blood. The potential markers that have been identified include tissue inhibitor of metalloproteinase-1 (TIMP1), chitinase 3-like 1 (YKL-40), galectin-3 (Gal-3), cytokeratin 19 (CK19), and angiopoietin-1 (Ang-1). A recent study by Makki et al. did not confirm differences in the levels of these markers in the serum of patients with benign and malignant thyroid changes [20]. Further studies on the potential markers of carcinogenesis in the thyroid gland are necessary.

A relatively new field of biological science is metabolomics, and it is a method that allows for quantitative measurements of overall metabolomic responses to pathophysiological or genetic changes in living organisms [21]. A schematic of this process is presented in Figure 2 [22].

It seems logical that the next step in the field of cell biology after the assessment of genome transcription products (mRNAs and proteins) is the study of the metabolome. This new manner of examining the processes taking place in living organisms may improve our understanding of the biochemistry of cancer and may help develop powerful diagnostic tools. In recent years, many studies have been published that assessed metabolite profiles using NMR spectroscopy and mass spectrometry in cancers, such as cancers of liver, kidney, ovary, breast, colon, esophagus, prostate and others [22-26].

\section{Metabolomics for the diagnosis of thyroid nodules}

Studies of the metabolome in thyroid diseases have been performed for approximately 20 years. Studies have used different analytical techniques and have focused on different compound groups. The sample collection methods used are also important. By definition, metabolomic studies allow for the use of samples of various origins, including tissue samples [27,28,37]; breath samples [29]; and biofluids,

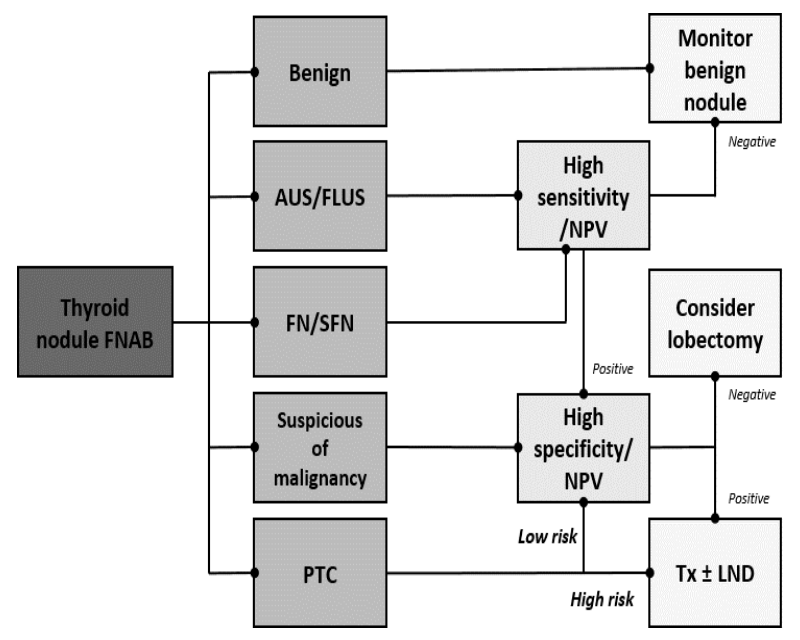

Figure 1: Algorithm for handling of thyroid nodules [17].

such as serum [28,30,31], urine [32], and saliva [33]. This allows metabolomic studies to purely use samples obtained in a less invasive manner (i.e., serum, urine, and saliva). In regards to thyroid cancer, metabolomic studies that have been conducted to date used multiple types of samples and analytical techniques $[27,28,34-39,41,43,44,47,53]$. Two basic strategies are used in metabolomics. The first is an untargeted approach, where all compounds are tested in a particular sample at a given time. The second is a targeted approach, which focuses on a specific group of compounds in sample. Both approaches are widely used [40].

The selected studies described below show that it is possible to use samples from different sources and to use different analytical techniques and different metabolomic strategies for diagnostic support for thyroid cancer. Such studies may facilitate the identification of changes in the thyroid gland.

\section{Magnetic resonance spectroscopy-based metabolomic studies of thyroid nodules}

In 1994, Delbridge et al. described differentiating thyroid cancer tissue from normal thyroid gland tissue using proton magnetic resonance spectroscopy with $100 \%$ sensitivity. Furthermore, they were able to distinguish two groups of benign adenomas, one with spectrum changes similar to those seen in healthy tissues and one with spectrum changes comparable to those in thyroid cancer tissues, based on the difference in the ratio of two NMR resonances regions, lipids at 1.7 $\mathrm{ppm}$ and amino acids at $0.9 \mathrm{ppm}$. In normal tissue, the ratio was above 1.1 , and in cancer tissue, it was below 1.1 [34].

Mackinnon et al. expanded on the studies of thyroid cancer performed by the Mountford group using NMR methods with the application of two-dimensional (COSY) spectra, and this allowed signals that overlapped on one-dimensional spectra to be distinguished from one another. Ninety-three samples from patients after subtotal or total thyroidectomy were used in that study, and the results indicated that the incidence of cholesterol/cholesteryl esters and di-/triglycerides cross peaks could be used to distinguish carcinomas from benign lesions [41].

Promising results were obtained by King et al. [42] and were similar to previously reported results [41]. In that study, 13 samples were examined by proton magnetic resonance spectroscopy. Eight samples 


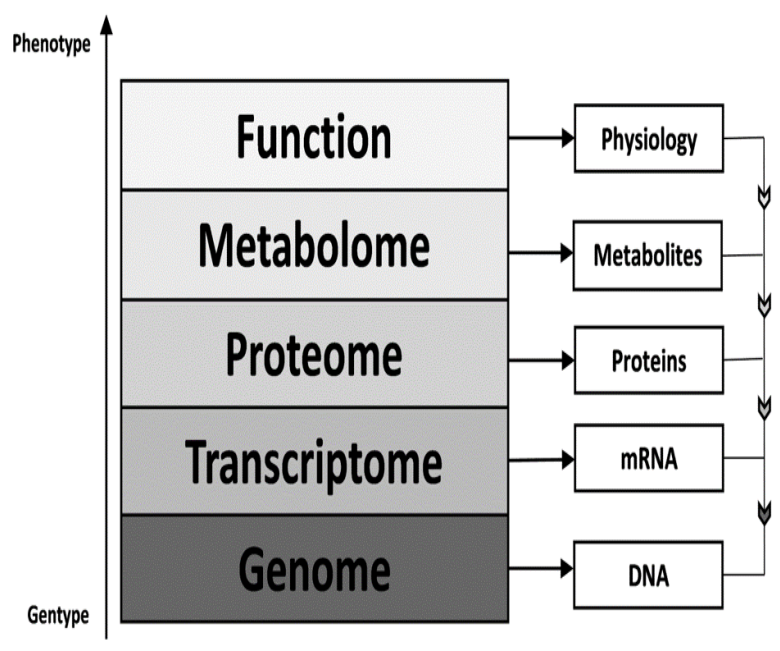

Figure 2: Schematic of the processes examined by 'omics' science [22].

were classified as thyroid cancers, and 5 samples were classified as healthy controls. The characteristic region of the spectrum originating from FA was shown to have diagnostic potential, and the presence of a choline signal also had diagnostic potential, with a high choline/ creatine ratio indicating cancerous tissue.

The findings of that study were confirmed by Gupta et al. who conducted an in vivo study involving analysis of the choline resonance signal by MR spectroscopy. A choline resonance signal was identified in all 8 of the cancer tissue samples analyzed in contrast to only 1 of the 17 benign tissue samples analyzed. This method proved to be $100 \%$ sensitive and $94.11 \%$ specific for the identification of thyroid cancer, which is in accordance with the results of King et al. [42]. The presence of a choline signal in the cells of a malignant tumor may be caused by an increased choline concentration due to rapid cancer cell proliferation [35].

Other studies have also demonstrated the possibility of using MRS as an alternative diagnostic method. However, in vivo MRS can result in inaccurate spectra, hindering the achievement of an accurate diagnosis due to the impacts of the surrounding fat, tumor size, tumor location, the act of the patient breathing and swelling near the tumor [44].

The results obtained in previous studies [34,35,41,42] were confirmed seventeen years later by Jordan et al. using ${ }^{1} \mathrm{HR}-\mathrm{MAS}$ NMR spectroscopy. They obtained a characteristic spectrum of papillary carcinoma that allowed for the differentiation of cancer, follicular adenoma and normal tissue, and this was confirmed histologically by FNA biopsy. As for the study described above, the distinction between the groups was based on the ratio of two NMR resonances - lipids at $1.7 \mathrm{ppm}$ and amino acid residues at $0.9 \mathrm{ppm}$. Although that study only examined 13 pairs of samples, it shows the possibility of the use of ${ }^{1}$ HRMAS in addition to FNA for the differentiation of follicular tumors [44].

The next studies that clearly demonstrated the usefulness of HRMAS-NMR in the diagnosis of thyroid tumors were performed by Torregrossa et al. [43]. They examined tissue samples from surgical biopsies of 72 patients. The study allowed for distinction of benign, malignant and normal tissue samples. The group of benign lesions included adenoma (A) and nodular goiter (GN), while the group of malignant lesions included anaplastic carcinoma (AC), papillary thyroid cancer (PTC) and follicular thyroid cancer (FTC). Multivariate data analysis (MDA) was performed, which allowed for the generation of predictive models, and the results were confirmed by ROC curve analysis, which revealed an AUC of 0.77 . Based on the OPLS-DA loadings, these studies revealed the set of essential metabolites that indicated changes in the thyroid gland. The most important changes in metabolites that indicated malignancy were increase in lactate and taurine and decreases in choline, phosphocholine, myo-inositol and scylloinositol. In this study, decreases in unknown compounds were also observed in the malignant group [43]. The observed changes in metabolite levels are associated with the Warburg effect [45] and other phenomena that affect metabolism during carcinogenesis [30,46,47].

The presented studies $[30,34,35,41,42,43,44]$ showed that among the metabolites that are possible biomarkers, those involved in lipid metabolism play a crucial role in carcinogenesis [48]. Therefore, lipidomics trends associated with carcinogenesis have been identified; however, while MS spectrometry is the method of choice for investigating lipids, NMR studies were also performed.

A targeted metabolomics approach was used by of Y. Yoshioka et al. to examine thyroid carcinogenesis, and they examined 61 tissue samples divided into 4 groups - control (27), papillary cancer (15), adenoma (13), and Basedow disease (6). ${ }^{1} \mathrm{H}$-NMR measurements were performed on lipids extracted using the Folch's method [49]. The compounds that had different levels between groups were dolichols, cholesterol, and cholines contained in phosphatidylcholine plus sphingomyelin (PC+SPH). The level of dolichols was lower in the cancer and Basedow groups but was normal in the adenoma samples. Cholesterol was only higher in the cancer samples. The level of acyl chain double bounds and the level of PC+SPH were only significantly higher in the cancer samples compared to the normal tissue samples. The results indicate a disturbance in the isoprenoid synthesis pathway in the papillary cancer samples [37], and this may related to a defect in a feedback mechanism that is controlled by the intracellular concentration of cholesterol [50].

Another group of compounds used for cancer identification and description in targeted metabolomics approaches is the phospholipids. The results of plasma ${ }^{31} \mathrm{P}$ NMR spectroscopy studies demonstrated the possibility of distinguishing between hypothyroid patients with cancer, hypothyroid patients in remission and healthy controls. The groups of hypothyroid patients with cancer have lower levels of PE+SM (phos phatidylethanolamine+sphingomyelin) and PC (phosphatidylcholine) than subjects in remission [30]. These changes in the levels of PE + SM and PC in patients with cancer may be caused by tumor metabolism, namely increased metabolism of phospholipids to allow for greater proliferation of tumor cells [38].

In our studies [27], tissue PBS extracts were used to compare groups of patients with different changes in the thyroid gland. The samples were divided into four groups - healthy control $(\mathrm{H})$, nonneoplastic nodules (NN), follicular adenoma (FA) and malignant thyroid cancer (TC). Using chemometric tools, differences between groups in the relative concentrations of specific metabolites were recognized. For all comparisons, OPLS-DA models were used. For two types of benign changes - FA and NN - the key changes observed were 
increased concentrations of the branched chain amino acids isoleucine and valine and decreased concentrations of citrate and $\mathrm{N}$-acetylated compounds in the FA group. In comparison FA and TC highest influence on separation had scyllo-inositol and myo-inositol together and also lower levels of methionine and lactate in relation to FA. The TC group was distinguished from the $\mathrm{NN}$ group based on differences in the levels of BCAAs, lactate, scyllo-inositol, myo-inositol, citrate and NACs.

Changes in metabolites levels in thyroid lesion can be transition point between healthy and development of thyroid cancer. The main differences observed between healthy tissue extracts samples and samples from thyroids altered in any way were lower levels of lipids, alanine, methionine, acetone, glutamate, glycine, lactate, tyrosine, phenylalanine and hypoxanthine, and the differences that were specific to cancerous thyroid tissues were lower levels of scyllo- and myo-inositol. The differences in those metabolites may be related to increased membrane biosynthesis, tumor development, the Warburg phenomenon and osmoregulation of cancer cells, indicating the possibility of cancer progression in the thyroid gland.

\section{Mass spectrometry-based metabolomic studies of thyroid nodules}

As previously mentioned there are two types of metabolomics approaches, untargeted and targeted. The main goal of metabolomics is to determine the levels of compounds in samples and the relationships of the increases and decreases in those levels with clinical conditions.

S Guo et al. [28] focused on the evaluation of lipidomic profiles in serum and tissue samples using MS. They obtained 289 serum samples and 36 tissue fragments. The samples were classified into 3 groups: healthy controls (serum-122; tissue-15), malignant thyroid MTC (serum-124, tissue-16) and benign thyroid - BTT (serum-43, the tissue-6). The goal of the study was to clearly distinguish the control group from the MTC group, the control group from the BTT group and the MTC group from the BTT group. The identification of compounds that were most valuable for differentiating the groups indicated that similar compounds were different between groups in serum and tissues. These compounds included PC (34:1) - (phosphatidylcholine), PC (36:1), PC (38:6), PA (36:2) - (phosphatidic acid), PA (36:3), PA (38:3), PA (38:4), PA (38:5), PA (40:5) and SM (34:1) - (sphingomyelin) [32]. Overexpression of FNAS (fatty acid synthase) and SCD1 (stearoyl-CoA desaturase-1) was observed in the serum and tissues samples from the MTC group. These enzymes can increase the levels of cancerogenic lipids, which are monounsaturated and saturated fatty acids. These metabolites can infiltrate the blood from tumor tissues via diffusion, which may have resulted in the increased level of these metabolites observed in the serum samples of cancer patients [28,51].

With the use of the relatively new technique of imaging mass spectrometry, IMS, Ishikawa $S$ et al. compared papillary cancer tissues (7 samples) to normal tissues with a focus on phospholipids. A small number of samples were analyzed, but this study proved that it is possible to use IMS as a metabolomic diagnostic tool. The study was able to distinguish between papillary cancer and normal tissue samples. The most important phospholipids that were found to have different concentrations between the two groups were phosphatidylcholine (16:0/18:1 and 16:0/18:2) and sphingomyelin (d18:0/16:1). These compounds are present in higher concentrations in papillary cancer then in healthy tissue [39]. The results also suggest overexpression of SCD1 in papillary cancer $[39,52]$.

A benefit of metabolomic studies is the ability to use samples collected less invasively than those collected via biopsy or surgery, and finding a marker or characteristic profile of metabolites that can be examined in material collected via less invasive approaches is highly desirable. For example, Yao et al. performed a metabolomic study mainly on serum samples. By examining changes in metabolite levels, they attempted to differentiate more global changes occurring in the thyroid gland. The study examined a total of 140 serum samples (30 papillary thyroid cancer, 80 - benign thyroid nodules, and 30 - healthy controls). Measurements were made using the LC-MS method. The results showed a general decline in the level of metabolites detected in the papillary thyroid cancer patients in relation to the patients with benign changes in the thyroid. Moreover, the two groups were distinguished from one another based on differences in the amount of FFA used in the biochemical reactions (most likely for beta-oxidation and the synthesis of ketone bodies) and the concentration of 3-hydroxybutyric acid, which is the intermediate product in fatty acid metabolism. In the future, differences in the levels of these metabolites may be used to distinguish patients with PTC from those with nodular goiter. However, the most important observations were the differences in the levels of related carnitines and the increased level of 3-hydroxybutyric acid in patients with papillary thyroid cancer; 3-hydroxybutyric acid can be considered a potential biomarker [36].

To further examine the use of samples collected using less invasive methods, L. Guo et al. examined exhaled breath samples. Volatile compounds present in the samples were measured using the GC-MS analytical technique. Ninety-six samples were used in the study and were divided into 3 subgroups: nodular goiter $(n=25)$, malignant nodule $(n=39)$ and control $(n=32)$. Based on MDA, including VIP values, groups of metabolites that could be used to differentiate the three groups were assigned to particular comparisons. For control vs. benign nodule, the most important metabolites were sulfurous acid, cyclohexylmethyl hexyl ester, isolongifolene-5-ol, 3,5-decadien-7yne, 6-tbutyl-2,2,9,9-tetramethyl, cyclohexanone, 4-hydroxybutyric acid, phenol, and 2,2-dimethyldecane. For control vs malignant, the most important metabolites were cyclohexanone, 4-hydroxybutyric acid, phenol, and 2,2-dimethyldecane, as for control vs benign nodule, but also ethylhexanol, ethyleneglycol mono vinyl ester, cyclopropane, and 1-bromo-1-(3-methyl-1-pentenylidene)-2,2,3,3-tetramethyl. The validation of both models showed AUC values of 1.00 with $100 \%$ sensitivity and specificity. For benign vs malignant, the most important metabolites were (3-Methyl-oxiran-2-yl)-methanol, cyclopentane, 1,1,3-trimethyl-3-(2-methyl-2-propenyl) and trans-2-Dodecen-1-ol. In that scenario, AUC value was 0.901 , with a sensitivity of $92 \%$ and a specificity of $82 \%$. The detected metabolites were related to changes in biochemical pathways involved in cancer metabolism, lipid metabolism regulation by thyroid hormone, and benzene and 4-hydroxybutanoic acid metabolism [53]. The results of this study confirm the changes in metabolites highlighted by Yao et al. [36] (Table 1). 
Citation: Wojtowicz W, Pawełka D, Balcerzak W, Młynarz P (2016) Metabolomics Methods as a New Diagnostic Tool for Thyroid Nodules. Metabolomics 6: 161. doi:10.4172/2153-0769.1000161

Page 5 of 6

\begin{tabular}{|c|c|c|c|c|}
\hline Publication & Technique & Important changes & Sample type & Amount $(n=)$ \\
\hline Delbridge et al. & MRS & Lipids $1.7 \mathrm{ppm}$ and $0.9 \mathrm{ppm}$ amino acids regions ratio & Tissue & 98 \\
\hline King et al. & MRS & Choline/Creatine ratio and FA & Tissue & 13 \\
\hline Mackinnon et al. & 2D ${ }^{1} \mathrm{H}$ MRS & cholesterol/cholesteryl esters and di-/triglycerides & Tissue & 93 \\
\hline Gupta et al. & MRS & Choline & Tissue & 8 \\
\hline Jordan et al. & ${ }^{1}$ HRMAS & Lipids $1.7 \mathrm{ppm}$ and $0.9 \mathrm{ppm}$ amino acids regions ratio & Tissue & 13 \\
\hline Toregrrossa et al. & ${ }^{1} \mathrm{HRMAS}$ & $\begin{array}{l}\text { Lactate, taurine, choline, phosphocholine, myo-inositol, scylloinositol } \\
\text { and unknown compounds }\end{array}$ & Tissue & 72 \\
\hline Yoshioka et al. & ${ }^{1} \mathrm{H}$ NMR & Dolichols, cholesterol, acyl chain double bounds, $\mathrm{PC}+\mathrm{SPH}$ & Tissue & 61 \\
\hline Raffelt et al & ${ }^{31} \mathrm{P}$ NMR & $\mathrm{PR}+\mathrm{SM}$ and $\mathrm{PC}$ & Plasma & 70 \\
\hline Deja et al. & ${ }^{1} \mathrm{H}$ NMR & $\begin{array}{l}\text { Alanine, methionine, acetone, glutamate, glycine, lactate, tyrosine, } \\
\text { phenylalanine, hypoxanthine, scyllo- and myo-inositol }\end{array}$ & Tissue & 64 \\
\hline S Guo et al & FT-ICR-MS & $\begin{array}{c}\mathrm{PC}(34: 1), \mathrm{PC}(36: 1), \mathrm{PC}(38: 6), \mathrm{PA}(36: 2), \mathrm{PA}(36: 3), \mathrm{PA}(38: 3), \mathrm{PA}(38: 4), \\
\mathrm{PA}(38: 5), \mathrm{PA}(40: 5) \text { and } \mathrm{SM}(34: 1)\end{array}$ & Tissue/serum & $36 / 289$ \\
\hline Ishikawa et al. & IMS, MS/MS & PC (16:0/18:1 and 16:0/18:2) and SPH (d18:0/16:1) & Tissue & 7 \\
\hline Yao et al & LC-MS & FFA and 3-hydroxybutyric acid & Serum & 140 \\
\hline L Guo et al. & GC-MS & Ethyl hexanol, phenol, 4-hydroxybutanoic acid & Exhaled breath & 96 \\
\hline
\end{tabular}

Table 1: Summary of results of metabolomic studies of changes in the thyroid gland $[27,28,34-39,41,43,44,47,53]$.

\section{Summary}

Metabolomics methods have great potential in supporting the diagnosis of thyroid lesions and differentiating between different changes to the thyroid gland The results summarized here confirmed the possible benefits that this field of science brings to medicine.

Assessment of metabolite profiles could be a cheaper alternative to molecula tests of biopsy tissues. The issue with the latter seems to be the small amoun of material collected during FNA and the need to fix the material in an alcoholic solution for cytological evaluation. The material obtained for metabolomic assessment should also be used for genetic testing, requiring an additional biopsy and freezing of the specimen in a short period of time. That limitation may not be a technical problem but is an inconvenience for patients, as they have to undergo two biopsies of the same tumor, which increases the risk of complications.

Efforts to use samples collected non-invasively or in ways that are less problematic and dangerous for the patient have led to metabolomics research as an alternative for basic diagnostics. It seems that the most important goal of this method is to be able to clearly separate between subjects with benign tumors, subjects with malignant tumors and healthy subjects. In addition, the use of metabolomics for analytical methods and the processing and analysis of spectra and chromatograms are also increasing. These factors may make it possible to obtain even better results and generate more accurate models of differentiating between subjects for use in certain diagnostic models.

In addition, the protocols used in metabolomics for sample preparation and measurements should be standardized to facilitate the implementation of the results obtained in studies conducted for diagnostic purposes.

\section{Acknowledgement}

This project was supported by Wroclaw University of Technology S40531/Z0303.

\section{References}

1. Dean DS, Gharib H (2008) Epidemiology of thyroid nodules. Best Pract Res Clin Endocrinol Metab 22: 901-911.

2. Aspinal SR, Ong SG, Wilson MS, Lennard TW (2013) How shall we manage the incidentally found thyroid nodule? Surgeon 11: 96-104.

3. Leenhardt L, Grosclaude P (2011) Epidemiology of thyroid carcinoma over the world. Ann Endocrinol (Paris) 72: 136-148.

4. Chen AY, Jemal A, Ward EM (2009) Increasing incidence of differentiated thyroid cancer in the United States, 1988-2005. Cancer 115: 3801-3807.

5. Davies L, Welch HG (2006) Increasing incidence of thyroid cancer in the United States, 1973-2002. JAMA 295: 2164-2167.

6. Boufraqech M, Patel D, Xiong Y, Kebebew E (2013) Diagnosis of thyroid cancer: state of art, Expert Opin Med Diagn 7:331-42.

7. Moon WJ, Jung SL, Lee JH, Na DG, Baek JH, et al. (2008) Benign and malignant thyroid nodules: US differentiation - multicenter retrospective study. Radiology 247: 762-770

8. Kim EK, Park CS, Chung WY, Oh KK, Kim DI, et al. (2002) New sonografic criteria for recommending fine-needle aspiration biopsy of nonpalpable nodules of the thyroid. AJR Am J Roentgenol 178: 687-691.

9. Maia FF, Matos PS, Silva BP, Pallone AT, Pavin EJ, et al. (2011) Role of ultrasound, clinical and scintigraphyc parameters to predict malignancy in thyroid nodule. Head Neck Oncol 3: 17.

10. Cantisani V, Lodise P, Grazhdani H, Mancuso E, Maggini E, et al. (2014) Ultrasound elastography in the evaluation of thyroid pathology. Current status. Eur J Radiol 83: 420-428. 
Citation: Wojtowicz W, Pawełka D, Balcerzak W, Młynarz P (2016) Metabolomics Methods as a New Diagnostic Tool for Thyroid Nodules. Metabolomics 6: 161. doi:10.4172/2153-0769.1000161

Page 6 of 6

11. Wang HL, Zang S, Xin XJ, Zhao LH, Li CX, et al. (2012) Application of Realtime Ultrasound Elastography in Diagnosis Benign and Malignant Thyroid Solid Nodules. Cancer Biol Med 9: 124-127.

12. Cibas ES, Ali SZ (2009) The Bethesda System for Reporting Thyroid Cytopathology. Am J Clin Pathol 132: 658-65.

13. Bose S, Walts AE (2012) Thyroid fine needle aspirate: a post Bethesda update. Adv Anat Pathol 19: 160-169.

14. Yang J, Schnadig V, Logrono R, Wasserman PG (2007) Fine-needle aspiration of thyroid nodules: a study of 4703 patients with histologic and clinical correlations. Cancer 111: 306-315.

15. Dincer N, Balci S, Yazgan A, Guney G, Ersoy R, et al. (2013) Follow-up of atypia and follicular lesion of undetermined significance in thyroid fine needle aspiration cytology. Cytopathology 24: 385-390.

16. Chen JC, Pace SC, Chen BA, Khiyami A, McHenry CR (2012) Yield of repeat fine-needle aspiration biopsy and rate of malignancy in patients with atypia of follicular lesion of undetermined significance: the impact of Bethesda System for Reporting Thyroid Cytopathology. Surgery 152: 1037-1044.

17. Xing M, Haugen BR, Schlumberger M (2013) Progress in molecular-based management of differentiated thyroid cancer. Lancet 381: 1058-1069.

18. Eszlinger M, Krogdahl A, Muenz S, Rehfeld C, Precht Jensen E, et al. (2014) Impact of molecular screening for point mutations and rearrangements in routine air-dried Fine Needle Aspiration samples of thyroid nodules. Thyroid 24: $305-313$

19. Yu S, Liu Y, Wang J, Guo Z, Zhang Q, et al. (2012) Circulating microRNA profiles as potential biomarkers for diagnosis of papillary thyroid carcinoma. $J$ Clin Endocrinol Metab 97: 2084-2092.

20. Makki FM, Taylor SM, Shahnavaz A, Leslie A, Gallant J, et al. (2013) Serum biomarkers of papillary thyroid cancer. J Otolaryngol Head Neck Surg 42: 16.

21. Nicholson JK, Lindon JC, Holmes E (1999) 'Metabonomics': understanding the metabolic responses of living systems to pathophysiological stimul via multivariate statistical analysis of biological NMR spectroscopic data. Xenobiotica 29: 1181-1189.

22. Roberts MJ, Schirra HJ, Lavin MF, Gardiner RA (2011) Metabolomics: a nove approach to early and noninvasive prostate cancer detection. Korean J Urol 52:79-89.

23. Vermeersch KA, Styczynski MP (2013) Applications of metabolomics in cancer research. J Carcinog 12:19.

24. Kumar V, Dwivedi DK, Jagannathan NR (2014) High-resolution NMR spectroscopy of human body fluids and tissues in relation to prostate cancer. NMR Biomed 27: 80-89.

25. Abbasi-Ghadi N, Kumar S, Huang J, Goldin R, Takats Z, et al. (2013) Metabolomic profiling of oesophago-gastric cancer: a systematic review. Eur J Cancer 49: 3625-3637.

26. Montrose DC, Zhou XK, Kopelovich L, Yantiss RK, Karoly ED, et al. (2012) Metabolic Profiling, a Noninvasive Aproach for the Detection of Experimental Colorectal Neoplasia. Cancer Prev Res (Phila) 5: 1358-1367.

27. Deja S, Dawiskiba T, Balcerzak W, Orczyk-Pawiłowicz M, Głód M, et al. (2013) Follicular Adenomas Exhibit a Unique Metabolic Profile. ${ }^{1} \mathrm{H}$ NMR Studies of Thyroid Lesions. PLoS ONE 8: e84637.

28. Guo S, Qiu L, Wang Y, Qin X, Liu H, et al. (2014) Tissue imaging and serum lipidomic profiling for screening potential biomarkers of thyroid tumors by matrix-assisted laser desorption/ionization-Fourier transform ion cyclotron resonance mass spectrometry. Anal Bioanal Chem 406: 4357-4370.

29. Bos LD, Weda H, Wang Y, Knobel HH, Nijsen TM, et al. (2014) Exhaled breath metabolomics as a noninvasive diagnostic tool for acute respiratory distress syndrome. Eur Respir J 44: 188-197.

30. Raffelt K, Moka, D, Süllentrop F, Dietlein M, Hahn J, et al. (2000) Systemic alterations in phospoholipid concetrations of blood plasma in patients with thyroid carcinoma: an in vitro 31P high-resolution NMR study. NMR Biomed 13: $8-13$

31. Kobayashi T, Nishiumi S, Ikeda A, Yoshie T, Sakai A, et al. (2013) A nove serum metabolomics-based diagnostic approach to pancreatic cancer. Cancer Epidemiol Biomarkers Prev 22: 571-579.

32. Nam H, Chung BC, Kim Y, Lee K, Lee D (2009) Combining tissue transcriptomics and urine metabolomics for breast cancer biomarker identification. Bioinformatics 25: 3151-3157.

33. Wang Q, Gao P, Wang X, Duan Y (2014) The early diagnosis and monitoring of squamous cell carcinoma via saliva metabolomics. Sci Rep 4:6802

34. Delbridge L, Lean CL, Russell P, May GL, Roman S, et al. (1994) Proton magnetic resonance and human thyroid neoplasia II: Potential avoidance of surgery for benign follicular neoplasms. World J Surg 18: 512-516.

35. Gupta N, Goswami B, Chowdhury V, Ravishankar L, Kakar A (2011) Evaluation of the role of magnetic resonance spectroscopy in the diagnosis of follicular malignancies of thyroid. Arch Surg 146: 179-182.

36. Yao Z, Yin P, Su D, Peng Z, Zhou L, et al. (2011) Serum metabolic profiling and features of papillary thyroid carcinoma and nodular goiter. Mol Biosyst 7 : 2608-2614.

37. Yoshioka Y, Sasaki J, Yamamoto M, Saitoh K, Nakaya S, et al. (2000) Quantitation by (1)H-NMR of dolichol, cholesterol and choline-containing lipids in extracts of normal and phathological thyroid tissue. NMR Biomed 13:377-383.

38. Carpinelli G, Canese R, Giannini M, Belardelli F, Podo F (1992) In vivo 31P MRS studies on experimental tumors treated with tumor necrosis factor. Acta Med. Rom 30: 295-299.

39. Ishikawa S, Tateya I, Hayasaka T, Masaki N, Takizawa Y, et al. (2012) Increased expression of phosphatidylcholine (16:0/18:1) and (16:0/18:2) in thyroid papillary cancer. PLoS One 7: e48873

40. Fiehn O (2002) Metabolomics-the link between genotypes and phenotypes Plant Mol Biol 48: 155-571.

41. Mackinnon WB, Delbridge L, Russell P, Lean CL, May GL, et al. (1996) Two-Dimensional Proton Magnetic Resonance Spectroscopy for Tissue Characterization of Thyroid Neoplasms. World J Surg 20: 841-847.

42. King AD, Yeung DK, Ahuja AT, Tse GM, Chan AB, et al. (2005) In vivo ${ }^{1} \mathrm{H}$ MR spectroscopy of thyroid carcinoma. Eur J Radiol 54: 112-117.

43. Torregrossa L, Shintu L, Nambiath Chandran J, Tintaru A, Ugolini C, et al (2012) Toward the reliable diagnosis of indeterminate thyroid lesions: a HRMAS NMR-based metabolomics case of study. J Proteome Res 11: 3317-3325.

44. Jordan KW, Adkins CB, Cheng LL, Faquin WC (2011) Application of magneticresonance-spectroscopy- based metabolomics to the fine-needle aspiration diagnosis of papillary thyroid carcinoma. Acta Cytol 55: 584-589.

45. Koppenol WH, Bounds PL, Dang CV (2011) Otto Warburg's contributions to current concepts of cancer metabolism. Nat Rev Cancer 11: 325-337.

46. Holub BJ (1986) Metabolism and Function of myo-Inositol and Inosito Phospholipids. Annu Rev Nutr 6: 563-597.

47. Tessem MB, Swanson MG, Keshari KR, Albers MJ, Joun D, et al. (2008) Evaluation of lactate and alanine as metabolic biomarkers of prostate cancer using ${ }^{1} \mathrm{H}$ HR-MAS spectroscopy of biopsy tissues. Magn Reson Med 60: 510-516.

48. Fiorenza AM, Branchi A, Sommariva D (2000) Serum lipoprotein profile in patients with cancer. A comparison with non-cancer subjects. Int J Clin Lab Res 30: 141-145.

49. Folch J, Lees M, Stanley GHS (1957) A simple method for the isolation and purification of total lipids from animal tissues. J Biol Chem 226: 497-509.

50. Hinson DD, Chambliss KL, Toth MJ, Tanaka RD, Gibson KM (1997) Posttranslational regulation of mevalonate kinase by intermediates of the cholesterol and nonsterol isoprene biosynthetic pathways. J Lipid Res 38: 2216-2223.

51. Hori S, Nishiumi S, Kobayashi K, Shinohara M, Hatakeyama Y, et al (2011) A metabolomic approach to lung cancer. Lung Cancer 74: 284-292.

52. Falvella FS, Pascale RM, Gariboldi M, Manenti G, De Miglio MR, et al. (2002) Stearoyl-CoA desaturase 1 (Scd1) gene overexpression is associated with genetic predisposition to hepatocarcinogenesis in mice and rats. Carcinogenesis 23: 1933-1936.

53. Guo L, Wang C, Chi C, Wang X, Liu S, et al. (2015) Exhaled breath volatile biomarke analysis for thyroid cancer. Translational Research. Transl Res 166: 188-195. 